\title{
Gestion traditionnelle et statut des ressources génétiques du sorgho (Sorghum bicolor L. Moench) au Nord-Ouest du Bénin
}

\author{
Antoine Abel MISSIHOUN ${ }^{1 *}$, Clément AGBANGLA ${ }^{1}$, \\ Hubert ADOUKONOU-SAGBADJA ${ }^{1}$, Corneille AHANHANZO ${ }^{1}$ et \\ Raymond VODOUHE ${ }^{2}$
}

\author{
${ }^{1}$ Département de Génétique et des Biotechnologies, Faculté des Sciences et Techniques (FAST), Université \\ d'Abomey-Calavi, O1BP 526, Cotonou, Benin. \\ ${ }^{2}$ Biodiversity International, Office of West and Central Africa, 08 BP 0931, Cotonou, Benin. \\ *Auteur correspondant, E-mail :missihoun_antoine@yahoo.fr; Tél: +229-95-56-56-84 /97-99-38-06.
}

\section{RESUME}

Le sorgho, Sorghum bicolor (L.) Moench, est une importante ressource alimentaire au Bénin. Sa culture est essentiellement pratiquée au centre et au nord du pays. Afin de mieux comprendre le niveau de diversité et les systèmes traditionnels de gestion et de conservation des variétés locales cultivées au Nord-Ouest du pays, une enquête basée sur une approche participative de recherche a été conduite dans 13 villages régulièrement distribués dans la zone. Au total, 45 variétés locales (61 noms vernaculaires) ont été inventoriées, présageant d'une grande diversité génétique du sorgho dans le milieu. Le nombre moyen de variétés locales inventoriées est de cinq par village et de deux par ménage. La distribution des variétés à travers la zone d'étude a été déterminée et les principaux facteurs y afférant étudiés. La couleur des grains, la durée du cycle végétatif ou la valeur marchande sont les plus importants critères utilisés par les producteurs pour la dénomination, l'identification, la classification et la sélection des variétés. Les systèmes traditionnels de sélection et de gestion des semences varient substantiellement d'un village à un autre et d'une ethnie à l'autre. Aussi, l'étudea-t-elle révélé l'existence dans certains villages d'une menace de disparition des variétés à cycle végétatif long et à grains blancs. Des efforts concertés devraient donc être déployés pour la valorisation et la préservation des ressources génétiques de cette céréale afin d'accroître sa production pour une meilleure sécurité alimentaire au Bénin.

() 2012 International Formulae Group. All rights reserved.

Mots clés : Bénin, diversité variétale, conservation, Sorghum bicolor.

\section{INTRODUCTION}

Le rôle crucial joué par les populations autochtones africaines dans la domestication et la conservation des plantes n'est plus à démontrer. Il a consisté depuis l'avènement de l'agriculture à la préservation et la protection des espèces et écotypes utiles (Tourte, 2005). La communauté scientifique internationale a officiellement reconnu l'importance des contributions des populations locales et surtout des agriculteurs dans la conservation des ressources génétiques pour le bien-être des populations présentes et futures (OUA, 2002). Cette reconnaissance institutionnelle se fonde principalement sur l'évidence que les agriculteurs, d'une part, au fil des siècles de 
domestication, de gestion et de sélection, ont façonné la diversité des plantes cultivées et d'autre part, le constat que cette action perdure dans le temps dans certaines parties du monde. En effet, dans les agro-systèmes traditionnels, les agriculteurs utilisent une large diversité d'espèces et de variétés locales. Cette diversité est souvent culturellement importante (Brush, 2000; Teshome et al., 2007 ; Barnaud, 2007). Aussi, certaines variétés locales ont-elles des utilisations uniquement spécifiques ou stratégiques. Cependant, si le rôle des agriculteurs est reconnu, la compréhension des processus mis en jeu et des facteurs sociaux, économiques et écologiques de cette dynamique évolutive des plantes domestiquées est encore fragmentaire et inégalement connue selon les espèces et les conditions agro-écologiques. La taxonomie locale reste dans cet ensemble, un préambule à la documentation des connaissances paysannes liées à la gestion de la diversité génétique des plantes cultivées. Des investigations faites sur plusieurs plantes ont confirmé cette importance. C'est le cas par exemple du manioc (Manihot esculenta Crantz) en Amazonie (Emperaire et al., 1998), de l'igname (Dioscorea spp.) (Dansi et al., 2000) et des légumes traditionnels (AchiganDako et al., 2011) au Bénin.

Le sorgho, Sorghum bicolor (L.) Moench, est l'une des céréales majeures dans l'alimentation humaine dans les régions tropicales et subtropicales de l'Afrique et de l'Asie (Doggett, 1988). Il est aussi utilisé comme culture fourragère pour l'alimentation animale (ICRISAT, 2008) et également dans l'industrie. Avec une production moyenne de 58,6 millions de tonnes par an et une superficie emblavée qui s'étend sur plus de 48 millions d'hectares, le sorgho est la cinquième céréale sur le plan mondial après le maïs (Zea mays), le riz (Oryza spp.), le blé (Triticum spp.) et l'orge (Hordeum spp.) (FAOSTAT, 2008).

En Afrique, le sorgho est cultivé dans une large ceinture qui s'étend de l'Atlantique à l'Ethiopie et à la Somalie, bordée par le Sahara au nord et la forêt équatoriale au sud. Cette zone couvre les parties sèches de l'Afrique occidentale, orientale et australe, où les précipitations sont trop fortes pour que la culture du mil pénicillaire (Pennisetum glaucum (L) R. Br.) se pratique et, par contre, trop faibles pour que celle du maïs se développe. En Afrique subsaharienne, le sorgho est la deuxième céréale, du point de vue des superficies couvertes et des tonnages produits, après le maïs (FAOSTAT, 2008).

$\mathrm{Au}$ Bénin, le sorgho est une céréale d'importance capitale pour l'agriculture et l'alimentation des populations locales. Avec une production qui avoisine 142000 tonnes pour une superficie totale emblavée de 146 000 hectares en 2009, le sorgho est la deuxième céréale après le maïs (MAEP/DPP, 2010). Par exemple, au Nord du pays, le sorgho occupe $40 \%$ des superficies emblavées en céréales juste derrière le maïs qui représente environ 44\%. L'essentiel de cette production est consommé localement, ce qui montre le rôle stratégique du sorgho dans la sécurité alimentaire des ménages (Kayodé et al., 2005). Les formes d'utilisation du sorgho sont diverses. En effet, le grain du sorgho entre dans la préparation de pâtes locales (dibou, sifanou, foura), de bouillies (koko, sorou, kamanguia) et de boissons locales (tchoukoutou, chakpalo) (Kayodé, 2006). Le grain de sorgho est également utilisé dans la fabrication de semoule ou de farine, selon ses caractéristiques.

Dans l'agriculture traditionnelle, les variétés locales constituent l'essentiel du matériel végétal utilisé. Elles sont anciennes et bien adaptées aux conditions pédoclimatiques difficiles dans lesquelles elles sont cultivées. Sur la plupart des céréales, y compris le sorgho, des études de documentation des savoirs, pratiques et systèmes traditionnels de gestion de la diversité ont été entreprises dans plusieurs zones de production et leurs impacts sur la conservation à la ferme analysés. Chez les Duиpa, un groupe ethnique du Nord Cameroun, une étude réalisée à un niveau local, a montré une grande diversité des variétés locales du sorgho au niveau d'un seul 
village et a démontré la dynamique de cette diversité aussi bien dans le temps que dans l'espace (Barnaud et al., 2007). En Ethiopie, une étude similaire a exploré la dynamique de la gestion des agriculteurs dans les environnements marginaux de production de sorgho et a montré son caractère non statique et ouvert, ses contraintes et limites. Cette étude a démontré clairement la nécessité de concilier, conservation $e x$-situ et conservation in situ dans la préservation des ressources génétiques des sorghos (Seboka et al., 2006). Il est donc important d'étendre de telles études dans d'autres aires de culture afin d'analyser beaucoup plus finement ou de généraliser les impacts de ces savoirs paysans, pratiques et systèmes traditionnels de gestion sur la conservation à long terme des ressources génétiques du sorgho et l'orientation des programmes de recherche en vue de son amélioration.

En matière de production agricole, les savoirs et pratiques paysannes liés à la gestion de la diversité génétique des variétés locales de cette spéculation restent très peu documentés au Bénin. Des études concernant la perception des agriculteurs dans la connaissance des variétés de sorgho et leur diversité génétique en relation avec les rapports potentiels entre les génotypes et leur convenance à la préparation de mets spécifiques ont été conduites dans trois communes du Nord-Bénin (Djougou, Toucountouna, Banikoara). Il ressort de ces études que les agriculteurs disposent d'un grand nombre de variétés locales selon les propriétés culinaires et les traits agronomiques en relation avec les spécificités de l'environnement et la demande des consommateurs (Kayodé et al., 2005). Cependant, outre la nécessité d'étendre les investigations dans les autres zones de production du pays, les aspects liés à la gestion en milieu paysan de ces variétés locales ne sont pas abordés. En effet, le niveau de diversité par village et par ménage, le mode de gestion au champ, la taxonomie locale, l'origine probable et les pratiques liées à la gestion des semences de ces variétés locales ne sont pas documentés. Or pour la définition d'une meilleure stratégie de conservation, de valorisation et d'amélioration des ressources génétiques du sorgho au Bénin, la compréhension du mode de gestion des premiers acteurs que sont les agriculteurs reste capitale.

La présente étude qui utilise une approche de recherche participative proche des réalités paysannes a pour objectifs de : (1) inventorier les variétés locales cultivées dans la zone d'étude, (2) décrire l'étendue et la distribution de la diversité variétale, (3) décrire la taxonomie locale des sorghos cultivés, et (4) comprendre le système traditionnel de gestion et de maintien de la diversité des variétés locales de sorgho en milieu paysan. Enfin, cette étude sera soutenue par des recommandations en matière d'implication des résultats pour la conservation et l'amélioration variétale au Bénin.

\section{MATERIEL ET METHODES \\ Zone d'étude et potentialités de production du sorgho \\ L'étude a été conduite dans le} département de la Donga au Nord-Ouest du Bénin, l'une des zones les plus productrices du sorgho dans le pays. Ce département est situé dans la zone agro-écologique semi-aride caractérisée par une pluviométrie incertaine et irrégulière avec une seule saison pluvieuse et une saison sèche qui dure plus de 5 mois (Adam et Boko, 1993). Administrativement, ce département est subdivisé en quatre districts ou communes (Bassila, Djougou, Ouaké et Copargo). C'est une région où les sols ferralitiques de plus en plus caillouteux deviennent inadaptés à la culture du Sud vers le Nord au fur et à mesure qu'on s'approche des montagnes de l'Atakora. Le département est habité principalement par quatre groupes ethniques (Yom, Lokpa, Anni et Koura) qui possèdent une grande tradition de la culture du sorgho, ce qui fait de celui-ci l'une des plus grandes régions de production de cette culture au Bénin. Les centres urbains (Bassila, Djougou et Copargo) restent cosmopolites où se rencontrent plusieurs ethnies du pays.

La production du sorgho, bien qu'irrégulière dans les quatre communes 
suivant les saisons, demeure relativement importante. De 2004 à 2007, elle est en moyenne de 11302 tonnes pour une superficie moyenne emblavée de 13005 ha et un rendement moyen de 0,870 t/ha (MAEP/DPP, 2010). Ce rendement reste très variable d'une campagne agricole à l'autre à cause des irrégularités des pluies.

\section{Choix des sites de collecte et traitement des données}

Treize villages sont sélectionnés sur la base de la production du sorgho, la distance géographique et l'ethnie majoritaire (Tableau 1 ; Figure 1) de manière à couvrir tout le département. Les données sont collectées à travers les différents sites au cours d'une prospection effectuée en Décembre 2010, selon les outils et techniques d'une approche de recherche participative basée sur des observations directes, des discussions libres, des entretiens de groupes et des entretiens individuels suivant un guide de questionnaire et des visites de champs tels que rapportés par Adoukonou-Sagbadja et al. (2006) et Dansi et al. (2010). Les entretiens sont conduits dans chaque village avec l'aide des traducteurs locaux. Dans tous les villages enquêtés, les autorités administratives et/ou locales (chefs village, rois et notables, délégués, conseillers, etc.) sont impliquées pour faciliter les rencontres avec les producteurs. La rencontre démarre par l'entretien de groupe. Les producteurs sont réunis sous l'autorité d'une personnalité locale. Les informations d'ordre général (nom de la commune, du village et le groupe ethnique) sont recueillies. Après une présentation succincte des objectifs du programme de recherche aux producteurs, ils sont invités à faire la liste de toutes les variétés locales (noms vernaculaires) encore cultivées ou non dans le village. Les échantillons sont ensuite collectés pour s'assurer de la présence effective des différentes variétés locales encore cultivées et pour régler sur place les difficultés de synonymie et d'homonymie de noms. A travers des entretiens de groupe, les informations détaillées concernant les descriptions morphologiques, agronomiques et culinaires (selon la perception paysanne) sont documentées. Les informations sur le cycle végétatif, l'origine des premières semences, les utilisations faites de chaque variété, les facteurs qui déterminent le maintien ou la disparition de chacune des variétés locales sont recueillies. La distribution et la superficie occupée par les différentes variétés dans les champs sont analysées suivant la méthode d'analyse des quatre carrés décrite par Brush (2000) et Tuan et al. (2003) et récemment utilisée par Dansi et al. (2010). Cette méthode d'évaluation est basée sur deux paramètres à savoir le nombre de ménages et la taille de superficie cultivée. Elle permet de classer toutes les variétés existantes en quatre groupes: (Q1) variétés cultivées par beaucoup de ménages sur de grandes superficies, (Q2) variétés cultivées par beaucoup de ménages sur de petites superficies, (Q3) variétés cultivées par peu de paysans sur de grandes superficies et (Q4) variétés cultivées par peu de paysans sur de petites superficies. Pour ce faire, les différentes variétés sont individuellement prises et évaluées par les paysans pour le premier paramètre (nombre de ménages). Pour ce paramètre, les paysans sont interrogés sur chaque variété si elle est cultivée par beaucoup ou peu de ménages. Le même processus est adopté pour l'évaluation de toutes les variétés pour le deuxième paramètre (taille de la superficie emblavée). En combinant les résultats de l'évaluation pour les deux paramètres, toutes les variétés sont classées dans les différents quadrants et immédiatement présentées aux paysans pour d'éventuels commentaires. Après cette classification, la discussion est ensuite menée en détail sur chaque variété avec pour but de comprendre le statut de chacune d'elles. Ici, les raisons qui justifient la culture de chaque variété par beaucoup ou peu de ménages et sur de grandes ou de petites superficies sont documentées. Enfin, le système traditionnel de gestion et de maintien des semences de ces variétés locales est documenté.

A la fin des entretiens de groupe, 10 à 15 paysans volontaires (essentiellement des hommes) sont choisis au hasard pour les entretiens individuels. Cette phase de l'enquête a pour but de documenter le niveau de la diversité variétale au niveau de chaque ménage et de comprendre les critères de préférence et de sélection d'une variété par un 
paysan, l'origine de ses semences, son expérience dans la culture du sorgho, les variétés de sorgho que cultivaient ses parents, les raisons qui font qu'il ne cultive plus certaines de ces variétés, sa volonté ou non de compléter lesdites variétés, le nombre de champs de sorgho dont il dispose, le mode de gestion de ses variétés aux champs (en association avec d'autres cultures, en culture mono- ou poly-variétale), la méthode de sélection des semences et comment elles sont conservées. Au total, 108 ménages ont été enquêtés. Les entretiens en groupes et individuels sont libres, ouverts et sans contrainte de temps tels que recommandés par Christinck et al. (2000).

Dans chaque village, des échantillons des variétés disponibles sont collectés. Ces échantillons sont des panicules apportées par différents paysans. Ils proviennent directement de leurs greniers ou de leurs champs. Les paramètres descriptifs utilisés pour documenter les échantillons collectés sont ceux relatifs au numéro d'accession, au nom de l'institution de collecte, au nom du collecteur, à la date de collecte, au nom vernaculaire et ethnique, les informations relatives à l'échantillonnage et à la localisation du site de collecte. Les données obtenues ont été analysées au moyen de statistiques descriptives (fréquence, pourcentage de réponses, moyenne) pour générer des résumés et des tableaux au niveau des différents villages ou groupes d'individus en utilisant le logiciel SAS (SAS Institut, 1996).

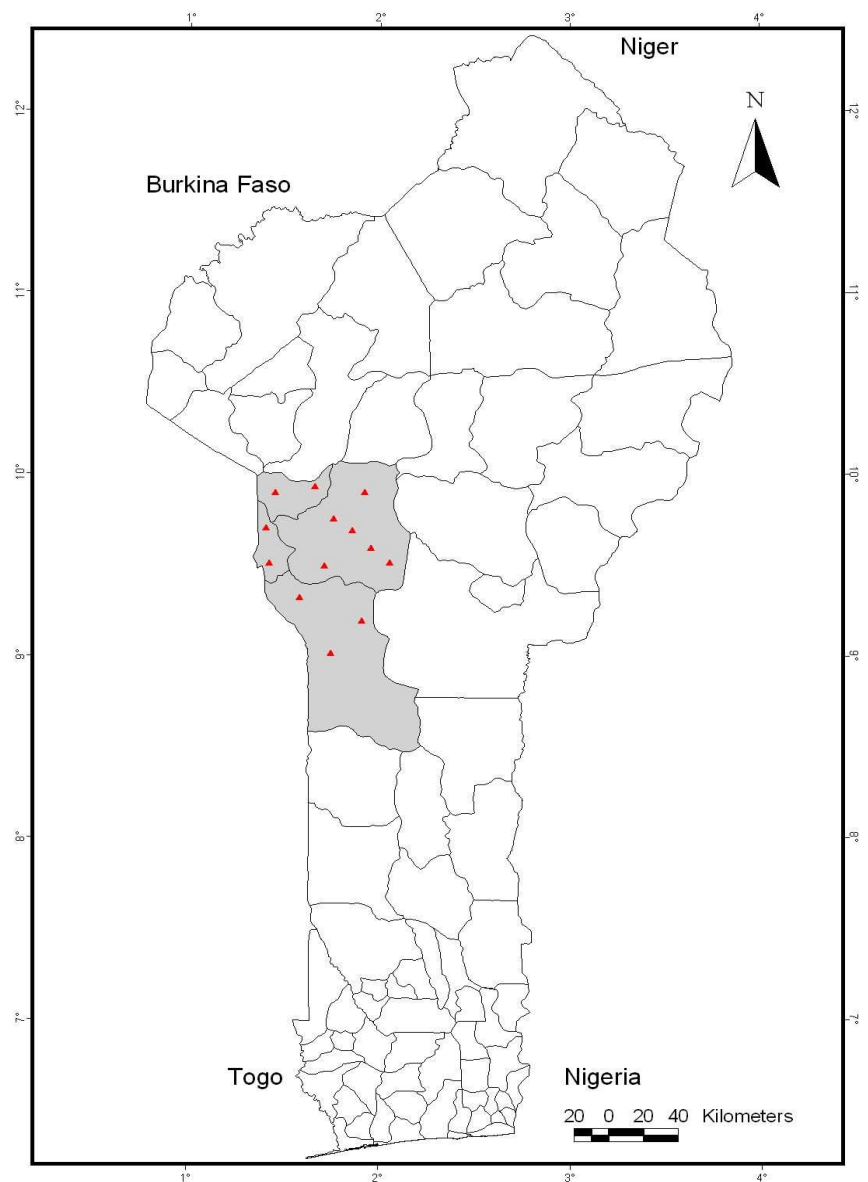

Figure 1 : Carte du Bénin montrant la localisation des villages sélectionnés et prospectés. 
Tableau 1: Nombre de villages enquêtés, leur localisation et le nombre de variétés inventoriées.

\begin{tabular}{lcccc}
\hline $\mathbf{N}^{\circ}$ & Villages & Communes & $\begin{array}{c}\text { Groupes } \\
\text { ethniques }\end{array}$ & $\begin{array}{c}\text { Nombre de variétés locales } \\
\text { inventoriées par village }\end{array}$ \\
\hline 1 & Salmanga & Bassila & Anni & 4 \\
2 & Guiguissou & Bassila & Anni & 4 \\
3 & Patargo & Bassila & Koura & 6 \\
4 & Darawinga & Djougou & Lokpa & 3 \\
5 & Sonaholou & Ouaké & Lokpa & 5 \\
6 & Kim-Kim & Ouaké & Lokpa & 3 \\
7 & Onklou & Djougou & Yom & 3 \\
8 & Danogou & Djougou & Yom & 10 \\
9 & Yoroussonga & Djougou & Yom & 9 \\
10 & Serou & Djougou & Yom & 7 \\
11 & Pélébina & Djougou & Yom & 4 \\
12 & Kpassabega & Copargo & Yom & 9 \\
13 & Singre & Copargo & Yom & 5 \\
& & & (Taneka) & \\
\hline
\end{tabular}

\section{RESULTATS}

Diversité variétale au niveau village et au niveau ménage

Au Nord-Ouest du Bénin, le nom générique du sorgho varie suivant le groupe ethnique. $Z o$ ou $Z a$ en Yom, il est appelé M'la en Lokpa, Aka en Koura et Ano ou Anoro en Anni (Tableau 2). Les critères de dénomination et d'identification des différentes variétés locales varient substantiellement et tiennent compte de la couleur des grains (principalement), de la forme des grains, de la forme d'ouverture des glumes, le nombre de grains par glumes, la forme des panicules, le lieu de culture, l'origine des premières semences.

Le nombre de variétés locales inventoriées par village varie de trois à dix avec une moyenne de 5,54 $\pm 0,69$ sur l'ensemble de la zone (Tableau 1). Les villages qui cultivent un nombre élevé de variétés locales sont habités majoritairement par l'ethnie Yom (3 à 10 variétés locales avec une moyenne de 6,71) et les villages qui détiennent un faible nombre de variétés locales sont ceux habités principalement par l'ethnie Lokpa (3 à 5 variétés locales avec une moyenne de 3,67) (Tableau 1).

La diversité des variétés locales au niveau de chaque ménage est faible et varie de 1 à 9 avec une moyenne de 2,06 $\pm 0,13$ sur l'ensemble des ménages prospectés. Les producteurs détenteurs d'un nombre élevé de variétés locales se retrouvent chez les Yom (jusqu'à 9 variétés locales).

Globalement, les variétés locales prospectées peuvent être regroupées en quatre groupes sur la base de la couleur des grains. Le premier groupe concerne les variétés à grains rouges. Elles sont actuellement les plus populaires et se retrouvent dans tous les villages prospectés. Elles représentent 21,33\% des échantillons collectés. D'introduction récente, elles résulteraient d'échanges entre paysans à partir du Togo voisin ou du Burkina Faso (la quasi-totalité des paysans de la zone de prospection reconnaissent que ces semences proviendraient du Nord-Ouest du pays par l'intermédiaire des peulhs nomades). Leur abondance actuelle s'expliquerait par leur relative tolérance à la sécheresse, leur précocité (cycle végétatif court), leur adaptabilité à des sols relativement pauvres et 
leur valeur marchande élevée (entre dans la préparation de Tchoukoutou, la bière locale largement consommée). Selon les producteurs enquêtés, elles seraient efficaces pour le traitement préventif de l'anémie.

Le deuxième groupe comporte les variétés à grains blancs. Elles représentent $30,66 \%$ des échantillons collectés. Elles sont les authentiques variétés locales ancestrales reconnues par l'ensemble des producteurs prospectés. Dans des conditions de pluviométrie et de sols adéquats, elles donneraient de meilleurs rendements que toutes les autres variétés. Elles sont bien farineuses et sont utilisées dans la confection des pâtes locales, plats de résistance habituels après le foufou d'igname dans la zone d'étude. Elles sont préférées par des ménages de grande taille (à nombre élevé de membres). Selon tous les producteurs enquêtés, ces variétés sont sensibles à la sécheresse et très exigeantes en matière de fertilité de sol. Néanmoins, elles sont préférées par les producteurs âgés qui n'ont plus la force physique d'emblaver de grandes superficies de champs qu'exigent les variétés du premier groupe.

Les variétés à grains de couleur intermédiaire constituent le troisième groupe. Elles sont morphologiquement les plus diversifiées. Elles représentent $41,33 \%$ des échantillons collectés et regroupent les variétés à grains roses, rougeâtres, blanchâtres, jaunâtres, noirâtres, blancs tachetés de pourpre ou de rouge, et bigarrés. Elles sont essentiellement semi-tardives ou tardives. D'après tous les producteurs enquêtés, elles sont relativement moins exigeantes en matière de pluviométrie et de fertilités de sol que les variétés du groupe précédent. Très appréciées pour leurs bons rendements en grains, elles sont néanmoins reconnues sensibles à la sécheresse. Comme les variétés du deuxième groupe, elles sont aussi utilisées dans la préparation des pâtes locales.

Le quatrième groupe concerne les variétés à grains jaunes. Elles représentent
6,66\% des échantillons collectés. Principalement cultivées pour être utilisées dans la pharmacopée traditionnelle, elles seraient efficaces, selon l'ensemble des producteurs enquêtés, pour le traitement préventif ou curatif du paludisme, des maux de ventre, des règles douloureuses et dans le traitement des femmes éprouvant des difficultés de conception. Elles sont utilisées dans l'alimentation des volailles, des boufs et d'autres petits ruminants domestiques dans le but de la prévention des pestes dévastatrices.

\section{Gestion au champ et distribution de la diversité des sorghos cultivés dans la zone d'étude}

Le mode de gestion au champ de la diversité des sorghos cultivés dans la zone d'étude reste la prédominance de la culture séparée des différentes variétés. Alors que la grande majorité des producteurs enquêtés $(85,39 \%)$ cultivent séparément les différentes variétés de sorgho dans des champs différents, avec $52,81 \%$ en culture pure (sans association avec d'autres cultures) et $32,58 \%$ en association avec d'autres cultures (maïs, le mil, niébé, arachide, etc.), seulement $14,61 \%$ des producteurs cultivent dans le même champ plusieurs variétés en mélange.

Un mode de gestion particulier des variétés locales de sorgho a été observé chez les Lokpa. En effet, cette ethnie distingue globalement, au sein de cette plante, deux groupes de variétés qu'elle cultive dans deux environnements strictement séparés. Les variétés dites sorgho de case, «Tia-m'la; Tia = case ou maison » cultivées aux alentours immédiats des habitations et des variétés dites sorgho de champ, «Talè-m'la; talè = champ» cultivées dans les grands champs loin des habitations.

$\mathrm{La}$ distribution et le niveau d'occupation des champs par les différentes variétés locales dans les différentes zones prospectées sont révélés par l'analyse des quatre carrés et sont intimement liés aux critères de préférence. Actuellement, les caractères qui prédominent dans le choix 
d'une variété dans l'ensemble des aires culturelles prospectées sont en premier lieu la précocité, la couleur de grain, la tolérance à la sécheresse, la facilité d'adaptabilité à des types de sols relativement pauvres et la flexibilité du temps de semis. Ainsi, les variétés Zomoaha, Zomoa, Zowémoha, Moussema, Koussèmèè, Anoro Kin'ka, Ano eranan chin' chin, Ano eranan, Aka kpankpan kékéré, Aka kpankpan lako qui constituent les variétés précoces ou intermédiaires à grains rouges (petits et gros grains) sont cultivées par beaucoup de ménages et sur de grandes superficies (Q1). Les variétés Agbani, Zopiha, Zokaram nini, Zogawa, Zobeundjoura, Zoniniléti, N'beumléti, Talèm'la (blanc), Aka foufou lako, Anoro foonon (Ano efoonon), Ano kaki, Kouhloumè qui sont des variétés à grains blancs ou à couleur intermédiaire, tardives ou semi-tardives et qui sont à haut rendement mais qui exigent une bonne pluviométrie et des terres fertiles sont actuellement cultivées par peu de producteurs (ceux qui disposent des terres appropriées) mais sur de grandes superficies (Q2) dans les zones où les niveaux de pluies sont encore acceptables. Autrefois, ces variétés occupaient le premier quadrant mais leur statut actuel serait dû aux irrégularités des pluies. «Lorsque les pluies manquent, on ne peut rien récolter» (déclaration de paysans interviewés). La variété sèmoutchè est cultivée par beaucoup de ménages et sur de petites superficies (Q3). Cette variété a une importance culturelle et sa culture est liée à deux fêtes traditionnelles identitaires chez les Lokpa (le Kamou ou la fête des chicottes et le Sann'kaalun une autre fête qui vient après la fête de kamou). Les variétés Moukoulikouté, Kpanchyan (Zo tétérrém, Kpatoni), Kassassahan, Zomoala (Zotirou), Zokpénaï, Moussii, M'sséé, Ano chochomi, Ano inodjo (Anoro manka), Vèmah sont cultivées par peu de ménages et sur de petites superficies (Q4). Celles-ci sont les plus menacées de disparition. Certaines variétés de ce groupe ont presque disparu de certains villages prospectés. C'est le cas de Kpanchyan, Kassassahan, Moukoulikouté (disparues de Kim-Kim), de M'sséé et Vèmah qui n'ont été échantillonnées qu'à Youroussonga, de Moussii qui n'a été collectée qu'à Pélébina. Les raisons évoquées pour cet abandon sont liées aux caractéristiques de ces variétés et parfois aux faits culturels. Par exemple, Kpanchyan est cultivée seulement pour ses feuilles rouges qui sont utilisées dans la pharmacopée traditionnelle et pour la teinture, Zomoala est utilisée en pharmacopée pour le traitement du paludisme et entre dans des préparations fermentées alors que la plupart des religions (en particulier la religion musulmane) interdisent la prise de boissons alcoolisées.

En général, dans la zone prospectée, les positions occupées par chaque variété locale sont liées à ses caractéristiques désirables et non désirables en relation avec les conditions édapho-climatiques.

\section{Taxonomie locale}

Dans cette étude, 61 noms vernaculaires ont été recensés et utilisés pour désigner (sous réserve de synonymie) 45 variétés locales de sorgho cultivées par les producteurs (Tableau 3). Les critères de dénomination et d'identification des variétés locales varient d'une ethnie à une autre et tient compte globalement de trois éléments : les critères liés à la description d'une ou plusieurs parties de la variété, les critères liés aux lieux de culture et les critères liés à l'origine supposée de la variété.

Chez les producteurs Yom, Koura et Anni, les critères liés à la description d'une ou plusieurs parties de la plante dominent et les critères utilisés se rapportent à la couleur, l'aspect ou la forme des grains, le nombre de grains par paire de glumes, la couleur ou le bâillement des glumes, la couleur des feuilles, la forme de la panicule. Ainsi, chez les producteurs Yom, nous notons les noms comme Zomoaha qui signifie sorgho rouge, Zomouara, sorgho rouge pur, Zopéha, Zopira ou Zopiha pour désigner le sorgho blanc, Sèm'gnim piha "sorgho à glumes blanches », Sèm'gnim moaha « sorgho à glumes rouges », 
Zokaram nini «sorgho dont les grains ressemblent aux yeux d'un oiseau appelé karam », Zoniniléti «sorgho ressemblant aux yeux bien ouverts », Zogawa «pas mûr, sorgho ressemblant à la couleur d'un fruit non mûr », Zo tétérém pour désigner le sorgho dont les feuilles rouges sont connues et vendues sous le nom de tétérém, Zobeumdjoura «sorgho dont la forme de panicule ressemble à la queue de cabri », beundjoura «sorgho dont la panicule ressemble à la queue d'un animal carnivore sauvage » M'ssée signifie «il a encore ajouté un grain » pour faire allusion au doublet de grains porté par chaque paire de glumes. On note également des descriptions lâches comme Zoga «sorgho même, sorgho vrai » ou $\mathrm{Za}$ pour désigner simplement «le sorgho» ou encore Agbani qui évoque le caractère « difficile à moudre des grains blancs ».

Chez les producteurs Koura, les descriptions se rapportent essentiellement à la couleur et la grosseur des grains. Ainsi Aka kpankpan Lako désigne « sorgho rouge à gros grains », Aka kpankpan kékéré « sorgho rouge à petits grains », Aka foufou Lako «sorgho blanc à gros grains », Aka foufou kékéré «sorgho blanc à petits grains ». On note également chez ces producteurs des noms de sorgho liés à l'origine supposée de la variété comme Aka inodjo «sorgho étranger » pour désigner un type de sorgho qui aurait été introduit par les pèlerins musulmans du retour de l'Arabie-Saoudite (Mecque). Cette même variété est désignée par les producteurs Anni par Ano manka. Chez les producteurs Anni, les critères de dénomination des variétés restent similaires à ceux utilisés par les producteurs Koura. Ainsi, Ano érana désigne «sorgho rouge », Ano érana chin'chin «sorgho rouge pur», Anoro kin'ka «sorgho rouge », Ano foonon ou Anoro éfoonon pour désigner «sorgho blanc », Ano kaki désigne un type de sorgho d'un blanc sale. Quant aux producteurs Lokpa, la taxonomie locale des variétés locales de sorgho reste particulière par rapport aux autres ethnies. Les Lokpa regroupent les variétés cultivées en deux grandes catégories: les «Tia m’la» qui signifie littéralement «sorghos de case» et les Talè m'la, les «sorghos du champ ». Les différentes variétés sont classées dans chaque catégorie par rapport aux critères descriptifs. Ainsi, on distingue, par exemple, des Tia m'la rouges comme Moussèma, koussèmè et Lissèma et des Tia m'la blancs comme kouhloumè.

\section{Origine, sélection et gestion des semences}

Les semences utilisées par les producteurs de la zone d'étude sont maintenues et gérées traditionnellement. Aucun système formel de distribution de semences n'a été noté. La majorité des producteurs prospectés $(83,15 \%)$ ont hérité leurs semences de leurs parents, $15,73 \%$ ont reçu le tout ou une partie de leurs semences auprès d'un ami et seulement 8,99\% des producteurs (étrangers ou du retour d'une aventure d'un pays voisin) ont acquis leurs semences par achat. Acheter des semences au marché est un fait rare pour les producteurs autochtones. Les échanges de semences sont plutôt fréquents lors des échecs de semis. Les systèmes traditionnels de sélection et de gestion des semences varient substantiellement d'un village/ d'une ethnie à l'autre.

La plupart des producteurs rapportent qu'ils sélectionnent aux champs leurs semences après la récolte. Ils sélectionnent les grosses panicules bien pourvues de grains sains, non attaquées ni par les maladies ni par les oiseaux. Après cette sélection, ils les attachent par des pédoncules réunis et les sèchent accrochées aux troncs d'arbres à la maison ou au toit des cases. Les panicules séchées sont conservées telles quel ou sont ensuite battues et les grains conservés. Aucun traitement chimique des semences avant la conservation n'a été rapporté par les paysans. Les grains sont parfois mélangés à de la cendre puis conservés dans différents types de conteneurs. Les types de matériels utilisés sont des calebasses à cou long et des anciens bidons d'insecticides de coton. Selon ce qui 
est rapporté par l'ensemble des producteurs, ce système traditionnel de stockage pourrait maintenir les semences viables pendant 6 à 10 ans. Enfin, dans les ménages, lorsque plusieurs variétés sont cultivées, les semences des différentes variétés sont toujours stockées séparément afin d'éviter des mélanges de variétés. Cette stratégie de gestion traditionnelle des semences est une très bonne pratique comme l'avaient déjà fait observer plusieurs auteurs sur diverses spéculations comme le fonio (Adoukonou-Sagbadja et al., 2006 ; Dansi et al., 2010) ou le niébé (Baco et al., 2008).

Tableau 2: Variation du nom générique du sorgho suivant le groupe ethnique.

\begin{tabular}{lll}
\hline Ethnies & Nom générique & Autre nom \\
\hline Anni & Ano & Anoro \\
Lokpa & M'la (Moula) & - \\
Koura & Aka & - \\
Yom & Zo & Za \\
\hline
\end{tabular}

Tableau 3 : Synthèse des noms vernaculaires et synonymes.

\begin{tabular}{|c|c|c|c|c|}
\hline $\mathbf{N}^{\circ}$ & Noms vernaculaires & Autres noms & $\begin{array}{l}\text { Groupe } \\
\text { ethnique }\end{array}$ & Signification des noms \\
\hline 1 & Agbani & - & Yom & Difficile à moudre \\
\hline 2 & Aka foufou kékéré & - & Koura & Sorgho blanc à petits grains \\
\hline 3 & Aka foufou lako & - & Koura & Sorgho blanc à gros grains \\
\hline 4 & Aka inodjo & - & Koura & Sorgho étranger \\
\hline 5 & Aka kpakpan kékéré & - & Koura & Sorgho rouge à petits grains \\
\hline 6 & Aka kpankpan lako & - & Koura & Sorgho rouge à gros grains \\
\hline 7 & Ano chochomi & - & Anni & Sorgho amère \\
\hline 8 & Ano eranan & - & Anni & Sorgho rouge \\
\hline 9 & Ano eranan chinchin & - & Anni & Sorgho rouge pur \\
\hline 10 & Ano kaki & - & Yom & Sorgho couleur kaki \\
\hline 11 & Anoro foonon & Ano efoonon & Anni & Sorgho blanc \\
\hline 12 & Anoro kin'ka & Ano kin'ka & Anni & Sorgho rouge \\
\hline 13 & B'mdjoura & Zobeumdjoura & Yom & Sorgho queue de cabri \\
\hline 14 & Kèmnin piha & - & Yom & - \\
\hline 15 & Kouhloumè & Tiam'la (blanc) & Lokpa & - \\
\hline 16 & Koulom & - & Lokpa & - \\
\hline 17 & Koussem & Kssèmèè & Lokpa & Sorgho rouge \\
\hline 18 & Kpanchyan & - & Lokpa & Sorgho à gaines foliaires rouge \\
\hline 19 & Lam néza & - & Yom & - \\
\hline 20 & Lam'za moaha & - & Yom & - \\
\hline 21 & Lamza & - & Yom & - \\
\hline 22 & Moukloukouyè & - & Lokpa & Sorgho jaune \\
\hline 23 & Moukoulikouté & - & Lokpa & Sorgho jaune clair \\
\hline 24 & Mousséé (M’sséé) & - & Yom & Il a encore ajouté un grain \\
\hline 25 & Moussema & M'ssema & Lokpa & Sorgho rouge pur \\
\hline 26 & Moussii & - & Yom & Sorgho jaune sale \\
\hline
\end{tabular}




\begin{tabular}{|c|c|c|c|c|}
\hline 27 & Narabeunzo & - & Yom & Sorgho de bouses de vache \\
\hline 28 & Sèmgnin moaha & - & Yom & Sorgho à glumes rouges \\
\hline 29 & Sèmgnin piha & - & Yom & Sorgho à glumes blanches \\
\hline 30 & Sèmoutchè & - & Lokpa & Sorgho blanc pur \\
\hline 31 & Talèm'la & - & Lokpa & Sorgho du champ \\
\hline 32 & Vèmah & - & Yom & - \\
\hline 33 & $\mathrm{Za}$ & - & Yom & Sorgho \\
\hline 34 & Zobomdjouha, & Zobeundjouha & Yom & $\begin{array}{l}\text { Sorgho queue d'un animal } \\
\text { carnivore sauvage }\end{array}$ \\
\hline 35 & Zogawa & - & Yom & $\begin{array}{l}\text { Pas mûr, sorgho ressemblant à } \\
\text { la couleur d'un fruit non mûr }\end{array}$ \\
\hline 36 & Zokaram nini & - & Yom & $\begin{array}{l}\text { sorgho ressemblant aux yeux } \\
\text { d'un oiseau appelé karam }\end{array}$ \\
\hline 37 & Zokpénaï & - & Yom & Sorgho jaune clair \\
\hline 38 & Zomoaha & $\begin{array}{l}\text { Zomooha, } \\
\text { Zoumboua }\end{array}$ & Yom & Sorgho rouge \\
\hline 39 & Zomoala & $\begin{array}{l}\text { Zotihou, } \\
\text { Zomoila, } \\
\text { Zomoora, }\end{array}$ & Yom & Sorgho jaune \\
\hline 40 & Zomouara & Zoumbouara & Yom & Sorgho rouge pur \\
\hline 41 & Zoniniléti & - & Yom & $\begin{array}{l}\text { Sorgho ressemblant aux yeux } \\
\text { bien ouverts }\end{array}$ \\
\hline 42 & Zooga & - & Yom & Sorgho même, sorgho vrai \\
\hline 43 & Zopiha & Zopéra, Zopira, & Yom & Sorgho blanc \\
\hline 44 & Zoténtérém & Kpatoni & Yom & Sorgho à feuilles rouges \\
\hline 45 & Zowémoha & - & Yom & Sorgho rouge pur petits grains \\
\hline
\end{tabular}

\section{DISCUSSION}

Diversité variétale du sorgho et sa gestion paysanne au Nord-Bénin

Les attributs sociologiques et culturels des communautés humaines sont connus comme des facteurs majeurs influençant substantiellement le niveau de diversité, la conservation et l'utilisation des ressources génétiques des cultures (Sam et al., 2006; Joseph et Antony, 2008). Au Bénin, le sorgho est l'une des cultures jouant traditionnellement un rôle capital dans l'agriculture et l'alimentation des populations locales. Des études récentes présageaient de l'existence d'un grand nombre de variétés locales du sorgho cultivé par les agriculteurs (Kayodé, 2006). La grande diversité variétale observée dans la présente étude (45 variétés locales inventoriées) confirme ce fait. De même, bien que la diversité variétale observée au niveau village et au niveau ménage dans cette étude soit relativement faible par rapport à celle signalée dans d'autres études similaires conduites au Ghana (Kudadjie, 2006), au Cameroun (Barnaud, 2007) ou au Burkina Faso (Barro-Kondombo et al., 2010), elle reste importante sur l'ensemble de la zone d'étude. Cette grande diversité génétique du sorgho au Bénin est un atout important pour les programmes d'amélioration génétique de cette culture dans le pays.

Cette étude a montré clairement que la production du sorgho au Bénin est de plus en plus dominée par des variétés à grains rouges et à cycle végétatif court au détriment des variétés à grains blancs et à cycle végétatif 
intermédiaire ou long. Contrairement à ce qui est observé au Burkina-Faso où ces variétés sont cultivées sur de petites superficies (Barro-Kondombo et al., 2010), elles sont cultivées sur de grandes superficies (car elles ont un rendement moyen) afin de compenser le déficit de production par la grandeur de la superficie cultivée. Elles demandent assez d'investissements physiques et sont majoritairement cultivées par des jeunes producteurs. Ce changement progressif du comportement des producteurs dans la zone d'étude peut s'expliquer d'une part, par l'irrégularité des périodes de pluies et de sécheresse marquée par la réduction sensible des temps de pluies et d'autre part par la valeur marchande élevée des variétés à grains rouges et à cycle végétatif court. Ce constat corrobore celui fait par Kudadjie (2006) dans la région Nord-Est du Ghana.

Les pratiques paysannes de gestion des semences (sélection, échanges et introductions de variétés, modes de conservation, etc.) et des variétés au champ telles observées et décrites dans la présente étude sont des pratiques largement connues et déjà rapportées sur diverses spéculations dans la sous-région ouest-africaine (vom Broke et al., 2003 ; Adoukonou-Sagbadja et al., 2006 ; Baco et al., 2008 ; Dansi et al., 2010 ; BarroKondombo et al. 2010). Néanmoins, la prédominance de la culture séparée des différentes variétés comme mode de gestion au champ de la diversité des sorghos cultivés par les producteurs dans la zone d'étude apparaît contraire à celle décrite chez les producteurs Duupa au Cameroun qui cultivent le sorgho surtout en mélange poly-variétal (Barnaud, 2007).

La valeur de la taxonomie paysanne dans la gestion et la conservation des ressources génétiques des cultures a été rapportée par plusieurs auteurs (Sambatti et al., 2001 ; McGuire, 2002 ; Adoukonou-
Sagbadja et al., 2006 ; Kudadjie, 2006 ; Barnaud, 2007). La reconnaissance des noms donnés aux variétés et une connaissance parfaite du système traditionnel de classification sont importantes car le nom local est l'unité de base que les producteurs utilisent dans la gestion et la sélection de ces ressources génétiques. Ce savoir-faire est largement connu pour avoir des conséquences sur le niveau de diversité génétique et l'évolution de la plante (vom Brocke et al., 2003 ; Adoukonou-Sagbadja et al., 2006 ; Barry et al., 2007). L'exemple de la variété Sèmoutchè est une indication palpable. Ce Tia-m'la est rencontré uniquement chez les Lokpa et cultivé seulement pour des raisons rituelles. C'est une variété dont la sortie des panicules annonce la fête de la chicotte (Kamou). Tant que cette fête existera, il aura toujours de Sèmoutchè. Au Nord-Est de l'Ouganda, les noms vernaculaires employés par les producteurs pour désigner des variétés locales de patate douce, ont fourni d'importantes informations sur la période de maturité, la production et autres données relatives au rendement. Une compréhension plus approfondie de ce système de classification des producteurs utilisant leur système de descriptions et d'appellations serait utile pour expliquer les conceptions et perceptions locales d'une variété et constituerait davantage un enjeu pour la recherche. Notons enfin ici que le mode particulier de gestion des variétés au champ observé chez les producteurs Lokpa pourrait avoir un impact sur la structuration génétique à cause de la séparation d'environnements de culture qui limite les flux de pollen entre les variétés de sorgho connu déjà comme une plante préférentiellement autogame (Ollitrault et al., 1997). L'impact de cette séparation spatiale de la culture des variétés sur les flux de gènes sera spécifiquement évalué à l'aide 
des analyses génétiques moléculaires pour vérifier cette hypothèse.

\section{Implications pour la conservation des} ressources génétiques du sorgho

La présente étude révèle l'état actuel de la diversité au niveau village et au niveau ménage des variétés locales de sorgho au Nord du Bénin. En général, les producteurs détiennent encore une diversité phénotypique appréciable de variétés locales. Mais il a été aussi largement rapporté par les producteurs qu'à cause des perturbations climatiques, les variétés à cycle végétatif long (tardives) sont progressivement abandonnées au profit des variétés à grains rouges précoces. Ces dernières seraient d'introductions récentes à partir du Togo et dominent actuellement la culture. Leur préférence par les producteurs est liée à leur précocité, leur relative tolérance à la sécheresse, la flexibilité de leur période de semis, leur valeur marchande élevée par rapport aux variétés à grains blancs. Autrefois, les producteurs préféraient les variétés à grains blancs pour leur rendement élevé et leur rôle dans l'alimentation mais la réduction de leur espace de culture actuel est liée à leur sensibilité à la sécheresse et leur non adaptation aux sols relativement pauvres. Des efforts de recherche dans le sens de la conservation et de la création variétale devraient permettre d'aboutir à des variétés réunissant une bonne productivité, de bonnes caractéristiques culinaires et la tolérance à la sécheresse.

D'autres variétés à faible rendement ou à caractéristiques culinaires peu appréciables (par exemples Mousséé, vèmah) ou encore à fonctions spécifiques comme thérapeutiques (Zo tétérém, Zotirou) sont soumises à un risque important de baisse de diversité génétique et ont presque disparu de certaines zones. Or, ces variétés pourraient disposer des gènes de tolérance à la sécheresse ou de résistance aux Striga asiatica, véritables plantes parasites qui détruisent les cultures de sorghos. Une action urgente est donc nécessaire pour la conservation ex situ de ces variétés dans les banques de semences nationales, régionales ou internationales.

Dans ce même ordre d'idée, nous préconisons qu'au moment où les variétés locales ne sont pas encore sujettes à un risque de disparition liée à l'introduction et la promotion de variétés améliorées, il est encore tant de compléter les collections existantes, de procéder à une caractérisation complète (agro-morphologique et génétique moléculaire) des collections en vue de la constitution d'une collection de base (core collection) et d'organiser une documentation efficace des données relatives à ces collections et leur mise à la disposition de la communauté scientifique nationale et internationale pour une utilisation judicieuse pour l'amélioration de la production. En termes de conservation des ressources génétiques, les variétés locales du groupe Q4 qui sont en disparition, nécessitent une action urgente de préservation et de conservation $e x$ situ. Nous préconisons à cet effet, la duplication de leurs semences dans les différents centres de recherche du pays et dans les banques de référence des instituts internationaux de recherche et dans la banque de conservation à perpétuité de Svalvard. Pour les variétés des groupes Q2 et Q3, des stratégies complémentaires de conservation (ex situ et on farm) pourraient se développer afin de mieux préserver leur diversité génétique. La valorisation ou la promotion des pratiques culturelles ou rituelles liées à certaines variétés, l'exemple de la fête des chicottes (Kamou) dans l'aire culturelle Lokpa, serait dans ce cadre un atout important pour la sauvegarde de certaines variétés locales comme sèmoutchè. Quant aux variétés 
$\mathrm{du}$ groupe Q1, une action urgente de conservation ne semble pas nécessaire.

\section{Conclusion}

Une forte diversité phénotypique de variétés locales de sorgho est maintenue et gérée par les populations de la Donga. Les différentes variétés sont nommées suivant des critères liés à la description d'une ou plusieurs parties de la variété, les critères liés aux lieux de culture et les critères liés à l'origine supposé de la variété. La distribution et le niveau d'occupation des champs par les variétés sont déterminés par leurs critères de préférences par les producteurs. Les variétés précoces à grains rouges tolérant la sécheresse dominent progressivement la culture au détriment des variétés tardives exigeantes en matière de fertilité de sols et sensibles à la sécheresse. Un risque important de réduction de diversité génétique apparaît au niveau de certaines variétés à faible rendement (par exemple le sorgho à grains gémellés, Mousséé) et à usages spécifiques (par exemple thérapeutique comme le sorgho à grains jaunes amers, Zomoala ou le sorgho colorant, Zotétérém). Ce constat révèle la nécessité de développement de stratégies de conservation et de valorisation de cette importante culture au Bénin. Des efforts de recherche pour une caractérisation agromorphologique et génétique moléculaire des variétés locales sont nécessaires afin de faciliter une gestion conservatoire efficiente et une exploitation durable de leurs ressources génétiques en amélioration.

\section{REMERCIEMENTS}

Ce travail a été financièrement soutenu par le Département de Génétique et des Biotechnologies de l'Université d'AbomeyCalavi, Bénin. Nous remercions très sincèrement $\mathrm{M}$. DOSSOUKPEVI R. et BIGUEZOTON A. pour leur contribution inestimable dans la réalisation de cette étude. Nos remerciements vont également au Dr FANDOHAN B. pour les critiques et commentaires du manuscrit et enfin, nos remarquables reconnaissances aux producteurs qui ont accepté partager avec enthousiasme et intérêt leurs connaissances avec nous.

\section{REFERENCES}

Achigan-Dako E, N'Danikou S, AssogbaKomlan F, Ambrose-Oji B, Ahanchede A, Pasquinin M. 2011. Diversity, geographical, and consumption patterns of traditional vegetables in sociolinguistic communities in Benin: implications for domestication and utilization. Econ. Bot., 65: $129-145$.

Adam S, Boko M. 1993. Le Bénin. Les éditions du Flamboyant/EDICEF; 96.

Adoukonou-Sagbadja H, Dansi A, Vodouhè R, Akpagana K. 2006. Indigenous knowledge and traditional conservation of Fonio millet (Digitaria exilis Stapf, Digitaria iburua Stapf) in Togo. Biodivers. Conserv., 15: 2379-2395.

Baco MN, Ahanchedé A, Bello S, Dansi A, Vodouhè R, Biaou G, Lescure JP. 2008. Evaluation des pratiques de gestion de la diversité du niébé (Vigna unguiculata) : une tentative méthodologique expérimentale au Bénin. Cahiers Agric., 17: 183-188.

Barnaud A, Deu M, Garine E, McKey D, Joly HI. 2007. Local genetic diversity of sorghum in a village in northern Cameroon: structure and dynamics of landraces. Theor. Appl. Genet., 114: 237248.

Barnaud A. 2007. Savoirs, pratiques et dynamique de la diversité génétique : le sorgho (Sorghum bicolor ssp. bicolor) chez les Duupa du Nord Cameroun. 
Thèse de doctorat, Université de Montpellier II, France, p. 230.

Barro-Kondombo C, Sagnard F, Chantereau J, Deu M, vom Brocke K, Durand P, Gozé E, Zongo JD. 2010. Genetic structure among Sorghum landraces as revealed by morphological variation and microsatellites markers in three agroclimatic regions of Burkina Faso. Theor. Appl. Genet., 10(120): 1511-1523.

Barry MB, Pham JL, Courtois B, Billot C, Ahmadi N. 2007. Rice genetic diversity at farm and village levels and genetic structure of local varieties reveal need for in situ conservation. Genet. Resour. Crop Evol., 54: 1675-1690.

Brush SB. 2000. Genes in the Field: on-Farm Conservation of Crop Diversity. Lewis Publishers: Boca Raton; 3-26.

Christinck A, Vom Brocke K, Kshirsagar KG, Weltzien E, Bramel-Cox PJ. 2000. Participatory methods for collecting germplasm: experiences with farmers in Rajasthan, India. Plant Genetic Resour. News L., 121: 1-9.

Dansi A, Mignouna HD, Zoundjihékpon J, Sangare A, Ahoussou N, Asiedu R. 2000. Identification of some Benin Republic's Guinea yam (Dioscorea cayenensis/ Dioscorea rotundata complex) cultivars using randomly amplified polymorphic DNA. Genet. Resour. Crop Evol., 47: 619-625.

Dansi A, Adjatin A, Adoukonou-Sagbadja H, Falade V, Yedomonhan H, Odou D, Dossou B. 2008. Traditional leafy vegetables and their use in the Benin Republic. Genet. Resour. Crop Evol., 8(55): 1239-1256.

Dansi A, Adoukonou-Sagbadja H, Vodouhè R. 2010. Diversity, conservation and related wild species of Fonio millet (Digitaria spp.) in the northwest of
Benin. Genet. Resour. Crop Evol., 9(57): 827-839.

Doggett H. 1988. Sorghum ( $2^{\text {nd }}$ edn). Longman Scientific and Technical: London.

Emperaire L, Pinton F, Second G. 1998. Gestion dynamique de la diversité variétale du manioc en Amazonie du Nord-Ouest. Nature, Sciences et Sociétés, 6(2): 27-42.

FAOSTAT. 2008. Concertation technique sur les bilans céréaliers et alimentaires dans les pays du cilss et en Afrique de l'Ouest (Accra), Rapport du Bénin.

ICRISAT. 2008. Sorghum. http://www.icrisat. org/sorghum/sorghum.htm.

Joseph JK, Antony VT. 2008. Ethnobotanical investigations in the genus Momordica L. in the Southern Western Ghats of India. Genet. Resour. Crop Evol., 55: 713-721.

Kayodé. 2006. Diversity, users' perception and food processing of sorghum: Implications for dietary iron and zinc supply. $\mathrm{PhD}$ Thesis, Wageningen University, Wageningen, p. 152.

Kayodé APP, Adégbidi A, Linnemann AR, Nout MJR, Hounhouigan DJ. 2005. Quality of farmer's varieties of sorghum and derived foods as perceived by consumers in Benin. Ecol. Food Nutr., 44: 271-294.

Kudadjie CY. 2006. Integrating science with farmer knowledge: Sorghum diversity management in northeast Ghana. $\mathrm{PhD}$ thesis, Wageningen University, Wageningen, p. 220.

MAEP/DPP. 2010. Annuaire statistique. MAEP/DPP.

McGuire S. 2002. Farmers' views and management of sorghum diversity in Western Harerghe, Ethiopia: Implications for collaboration with formal breeding. In Collaborative Plant Breeding: Integrating Farmers' and Plant Breeders' 
Knowledge and Practice, Cleveland D, Soleri D (eds). Commonwealth Agricultural Bureaux International (CABI): Wallingford; 107-136.

Ollitrault O, Nyer JL, Chantereau J, Glaszmann JC. 1997. Structure génétique et dynamique des variétés traditionnelles de sorgho au Burkina Faso. In Gestion des Ressources Génétiques des Plantes en Afrique des Savanes, Begic A (ed). Solagral: Bamako, Mali; 231-240.

OUA. 2002. Projet Modèle Loi Nationale sur la Sécurité en Biotechnologie p.44.

Sam PM, Mohandas A, Shareef SM, Nair GM. 2006. Biocultural diversity of the endemic 'wild jack fruit tree' on the Malabar coast of South India. Ethnobot. Res. Appl., 4: 25-40.

Sambatti JBM, Martins PS, Ando A. 2001. Folk taxonomy and evolutionary dynamics of cassava: a case study in Ubatuba, Brazil. Econ. Bot., 55: 93-105.

SAS. 1996. SAS/STAT user's guide, Release 6 12. SAS Institute, Cary.

Seboka B, Hintum van T. 2006. The dynamics of on-farm management of sorghum in
Ethiopia: Implication for the conservation and improvement of plant genetic resources. Genet. Resour. Crop Evol., 5(53): 1385-1403.

Teshome A, Patterson D, Asfew Z, Torrance KJ, Arnason JT. 2007. Changes of Sorghum bicolor landrace diversity and farmers' selection criteria over space and time, Ethiopia. Genet. Resour. Crop Evol., 6(54): 1219-1233.

Tourte R. 2005. Histoire de la Recherche en Afrique Tropicale, aux Sources de l'Agriculture Africaine : de la Préhistoire au Moyen-âge (vol. 1). FAO; 34.

Tuan HD, Hue NN, Sthapit BR, Jarvis DI. 2003. On-farm management of agricultural biodiversity in Vietnam. Proceedings of a symposium. International Plant Genetic Resources Institute, Rome, Italy.

Vom Brocke K, Christinck A, Weltzien ER, Presterl T, Geiger HH. 2003. Farmer's seed systems and management practices determine pearl millet genetic diversity patterns in semiarid regions of India. Crop Sci., 43: 1680-1689. 